\title{
FAR INFRARED COHERENT SYNCHROTRON EDGE RADIATION AT ANKA
}

\author{
A.-S. Müller, I. Birkel, B. Gasharova, E. Huttel, R. Kubat, Y.-L. Mathis, D.A. Moss, W. Mexner, \\ R. Rossmanith, M. Wuensch, P. Wesolowski, ANKA-ISS, Forschungszentrum Karlsruhe, Germany, \\ F. Pérez, M. Pont, ALBA Synchrotron Light Source, Spain, \\ C.J. Hirschmugl, University of Wisconsin, Milwaukee, USA
}

\begin{abstract}
A synchrotron radiation source emits coherent infrared (IR) radiation when the electron bunch length is comparable to the wavelength of the emitted radiation (see for example $[1,2])$. To generate coherent radiation in the far IR (THz) region, a 'low alpha mode' has been devised at the ANKA storage ring operating at $1.3 \mathrm{GeV}$. The corresponding lattice has a significantly reduced momentum compaction factor. The spectral dependence of the emitted radiation is recorded at the ANKA-IR beam line, where the synchrotron light is produced in the fringe field of a bending magnet [3]. This edge radiation has the advantage of being more collimated than constant field radiation. This allows the observation of frequencies down to $1 \mathrm{~cm}^{-1}$ through a modest vertical aperture, which would not be possible with classical constant field emission due to the increasing beam divergence with decreasing frequency. The onset of coherent emission is found at a synchrotron frequency of about $10 \mathrm{kHz}$. At $5 \mathrm{kHz}$, an intensity enhancement of up to 5 orders of magnitude, with respect to the incoherent emission, is observed in the spectral range between 1 and $65 \mathrm{~cm}^{-1}$.
\end{abstract}

\section{INTRODUCTION}

The storage ring of the ANKA synchrotron radiation source is located at Forschungszentrum Karlsruhe in Germany. It is routinely operated at a beam energy of $2.5 \mathrm{GeV}$. The beam current is accumulated at $0.5 \mathrm{GeV}$ and then ramped to the end energy. This offers the opportunity to run the storage ring at any energy in the range between 0.5 and $2.5 \mathrm{GeV}$.

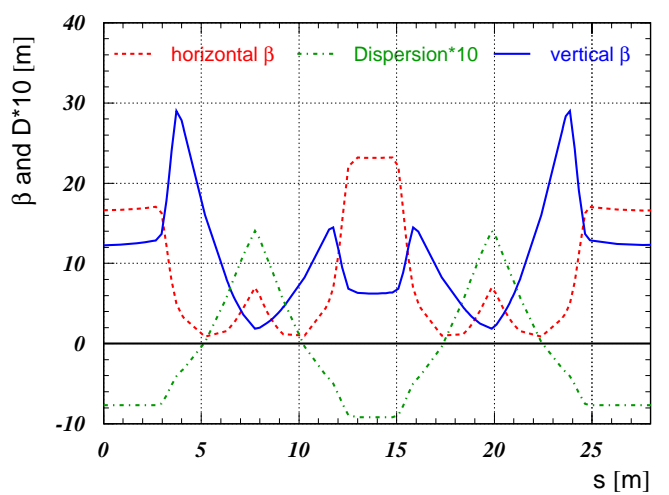

Figure 1: Betatron functions and horizontal dispersion of the low alpha optics for one of four sectors of the ANKA storage ring.
Coherent synchrotron radiation is generated when the electron bunch length is comparable to the wavelength of the emitted radiation [1,2]. Since the emission of long wavelengths is suppressed by shielding effects of the vacuum chamber, the bunch length needs to be sufficiently short in order to yield observable radiation. The flexibility in the choice of beam energy is of advantage for the generation of such short electron bunches. Previous studies have shown that the bunch length at ANKA is shortest at a beam energy of about $1.3 \mathrm{GeV}$ due to longitudinal instabilities at lower beam energies [4]. To further reduce the bunch length, the storage ring optics is modified to yield a lattice with a significantly reduced momentum compaction factor.

\section{THE DEDICATED LOW- $\alpha$ OPTICS}

The momentum compaction factor is defined as the relative increase of the electron's path length $C$ along the closed orbit with a momentum deviation $\Delta p$ with respect to the momentum of the reference particle $p_{0}, \Delta C / C=\alpha_{c} \Delta p / p_{0}$. Decreasing $\alpha_{c}$ will reduce the bunch length proportional with $\sqrt{\alpha_{c}}$. A reduction of $\alpha_{c}$ from the regular value of $7.2 \cdot 10^{-3}$ to a value of $1.4 \cdot 10^{-4}$ (corresponding to a bunch length of $\sigma_{s}=(0.86 \pm 0.05) \mathrm{ps}$ as derived from an interferogram for a bunch current of $I_{\text {bunch }}=0.35 \mathrm{~mA}$ ) was achieved but does not seem to be the ultimate limit. A further reduction will be tried out in future accelerator studies. Figure 1 shows the optics functions of the dedicated low$\alpha$ optics for one of the four sectors of the ANKA storage
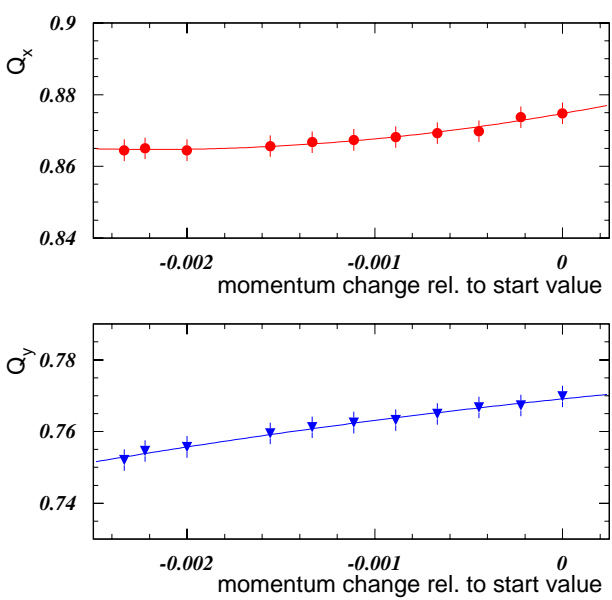

Figure 2: Measured horizontal (top) and vertical (bottom) tunes as a function of relative momentum change. 


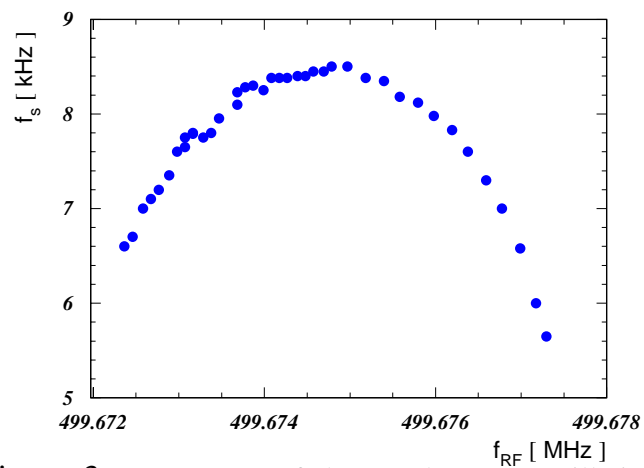

Figure 3: Frequency of the synchrotron oscillation's dipole mode measured with a strip-line as a function of the frequency of the RF system.

ring. Since $\alpha_{c}=1 / L \oint d s(D(s) / \rho(s))$, the reduction of the momentum compaction factor is achieved by rendering the dispersion partially negative with the help of the quadrupole family acting as "field lens" while at the same time using a focusing and a defocusing family to keep the tune variation reasonable.

Detailed studies have been carried out for an optics version with moderately reduced momentum compaction factor, not far away from the threshold of observation of coherent synchrotron radiation. Figure 2 shows a measurement of the horizontal and vertical chromaticities which are found to be $Q_{x}^{\prime}=9$ and $Q_{y}^{\prime}=5$ in agreement with the expectation from model calculations.

The momentum compaction factor can be expressed as a function of the momentum deviation in the following way:

$$
\alpha_{c}=\alpha_{0}+\alpha_{1} \frac{\Delta p}{p_{0}}+\alpha_{2}\left(\frac{\Delta p}{p_{0}}\right)^{2}+\ldots
$$

To determine the higher order terms, the frequency of the dipole mode of the synchrotron oscillation is measured with a strip-line as a function of the RF frequency (Fig. 3). This study was done for a momentum compaction factor slightly below the onset of the coherent emission. The momentum dependence of $\alpha_{c}$, derived from this measurement, is displayed in Fig. 4. The curve is a fit to the measurements and yields the values for the higher order terms of the momentum compaction factor. The leading coefficients

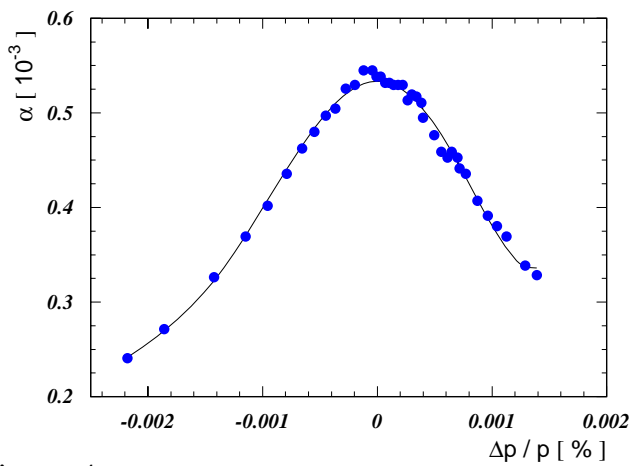

Figure 4: Momentum compaction factor derived from the measurement shown in Fig. 3 as a function of momentum deviation.

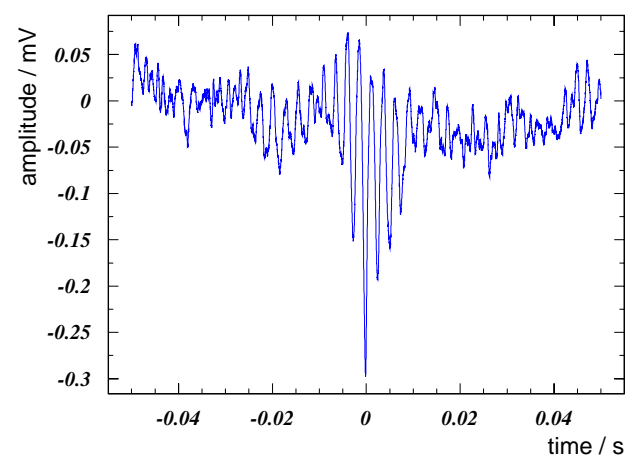

Figure 5: Bolometer signal as a function of time. The burst of coherent radiation is clearly visible. A stable emission would show as a signal constant in time.

are $\alpha_{0}=(0.533 \pm 0.002) \cdot 10^{-3}, \alpha_{2}=-(185 \pm 5), \alpha_{1}$ is found to be compatible with zero.

\section{BURSTING AND STABLE EMISSION}

Effects like beam instabilities and micro bunching due to the coherent synchrotron radiation itself act on the charge distribution, thus changing the bunch length [5]. Depending on the exact shape of the charge distribution and the bunch current, the mode of emission changes. For higher bunch currents a 'bursting' emission will dominate, below a certain threshold current, a steady emission is observed [6].

The emitted radiation in the far IR is detected at the ANKA-IR beam line [3], where the synchrotron light is produced in the fringe field of a bending magnet. This edge radiation has the advantage of being more collimated than constant field radiation. This allows the observation of frequencies up to $30 \mathrm{GHz}$ through a modest vertical aperture, which would not be possible with classical constant field emission due to the increasing beam divergence with decreasing frequency. The detector used for the studies is a liquid He cooled Si bolometer. The spectral dependence is recorded using a Bruker IFS66v/S spectrometer equipped with Mylar beam splitters with thicknesses between 6 and $125 \mu \mathrm{m}$ depending on the application. Figure 5 shows

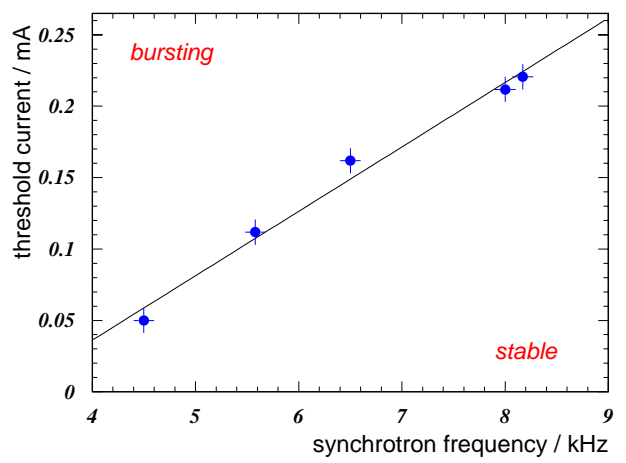

Figure 6: Bunch current at the threshold between bursting and stable emission for different values of the momentum compaction factor which is represented by the synchrotron frequency. 


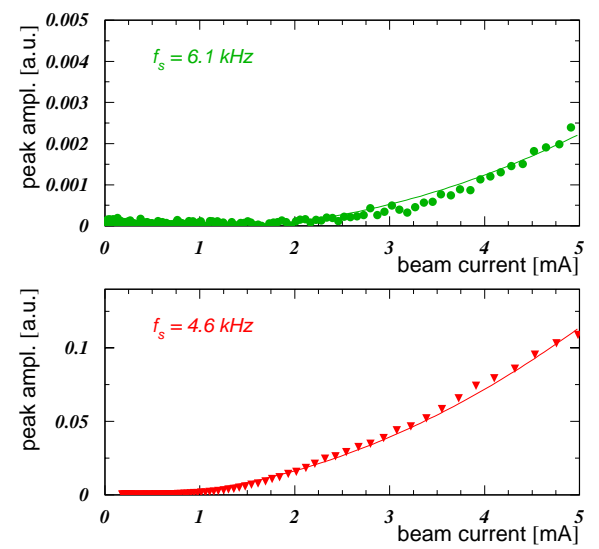

Figure 7: Peak amplitude of the interferogram as a function of beam current for different values of the synchrotron frequency. The curves are quadratic fits.

the bolometer signal measured as a function of time at the ANKA-IR beam line for a bunch current of $0.5 \mathrm{~mA}$. The trigger condition selecting the highest signal intensity allows to clearly see the evolution and decay of a burst of powerful radiation over a period of about $10 \mathrm{~ms}$ (about one longitudinal damping time). The threshold current for the transition from bursting to stable emission was determined for different values of the momentum compaction factor. The results are displayed in Fig. 6 where $\alpha_{c}$ is represented by the corresponding synchrotron frequency.

\section{FIR INTENSITY AND SPECTRUM}

In the case of the emission of coherent radiation, the amplitudes of the electromagnetic fields add up linearly, resulting in a quadratic enhancement of the radiation's intensity. The total power radiated by a bunch consisting of $N$ particles can be expressed as

$$
P_{\text {total }}=N P_{\text {incoherent }}\left(1+N f_{\lambda}\right)
$$

where $f_{\lambda}$ is a form factor describing the effect of the longitudinal charge distribution in the bunch. For a Gaussian charge distribution with RMS length $\sigma_{s}$ the form factor is given by $f_{\lambda}=\exp \left(-\left(2 \pi \sigma_{s} / \lambda\right)^{2}\right)$. This shows that the radiated intensity for a given wavelength will increase with decreasing bunch length. Figure 7 displays measurements of the Michelson interferogram peak amplitude as a function of total beam current for different bunch lengths. Both clearly show a quadratic dependence on the beam current with a significantly higher peak amplitude for the measurements with shorter bunch lengths.

The FIR intensity as a function of the wavenumber is shown in Fig. 8 for different synchrotron frequencies. The red curve represents the incoherent spectrum, the blue and green curves are measurements of the coherent spectrum. The FIR spectrum for short bunches covers the wavenumber range from 65 down to $1 \mathrm{~cm}^{-1}$. The intensity amplitfication due to the coherent emission is shown in Fig. 9 for a spectral range less sensitive to systematic effects (e.g. beam splitter cut-offs). Even for a synchrotron frequency of $5.0 \mathrm{kHz}$ (lower frequencies have been used during the

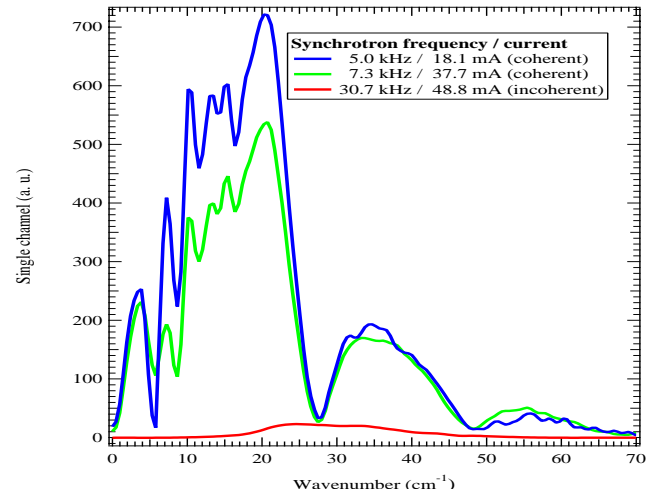

Figure 8: Measurements of the coherent and incoherent FIR spectrum with a $50 \mu \mathrm{m}$ Mylar beam splitter for different values of the synchrotron frequency.

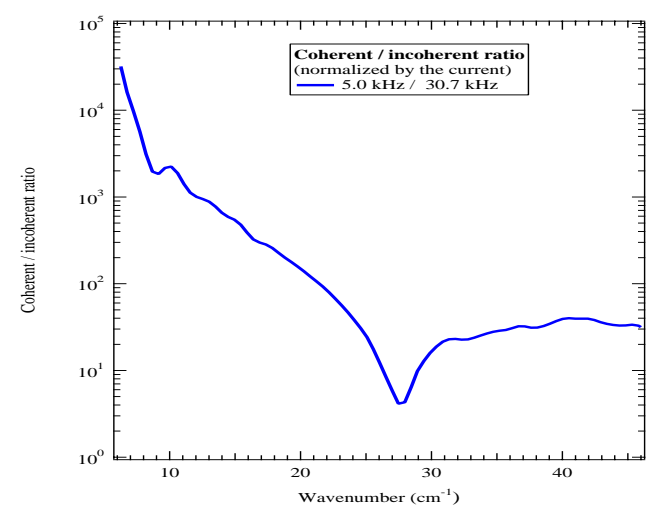

Figure 9: Ratio between the coherent and the incoherent FIR intensity.

studies) the intensity enhancement is almost 5 orders of magnitude.

\section{ACKNOWLEDGEMENTS}

Sincere thanks to all who have contributed to the results presented here. Especially we would like to thank K. Holldack, P. Kuske and G. Wüstefeld for interesting and instructive discussions and R. Stricker and M. Süpfle for technical support.

\section{REFERENCES}

[1] G.L. Carr et al. Investigation of Coherent Emission from the NSLS VUV Ring. PAC 1999.

[2] M. Abo-Bakr et al. Coherent mm-Radiation Experiments at the BESSY II Storage Ring. EPAC 2000.

[3] Y.-L. Mathis, B. Gasharova, D. Moss. Terahertz radiation at ANKA, the new synchrotron light source in Karlsruhe. In Journal of Biological Physics 29, pages 313-318, 2003.

[4] F. Perez et al. Beam Size and Bunch Length Measurements at the ANKA Storage Ring. PAC 2003.

[5] M. Abo-Bakr et al. Coherent Emission if Synchrotron Radiation and Longitudinal Instabilities. PAC 2003.

[6] F. Sannibale et al. A Model Describing Stable Coherent Synchrotron Radiation in Storage Rings. In Phys. Rev. Lett. 93 , number 9, 2004. 